\title{
Latent variable analysis indicates that seasonal anisotropy accounts for the higher prevalence of left-handedness in men
}

\author{
Ulrich S. Tran ${ }^{a, *}$, Stefan Stieger ${ }^{a, b}$ and Martin Voracek ${ }^{a}$ \\ ${ }^{a}$ University of Vienna, School of Psychology, Vienna, Austria \\ ${ }^{\mathrm{b}}$ Research Methods, Assessment, and iScience, Department of Psychology, University of Konstanz, Germany
}

A R T I C L E I N F O

Keywords:

Handedness

Laterality

Seasonal variation

Seasonal anisotropy

Latent class analysis

\begin{abstract}
A B S T R A C T
According to the Geschwind Galaburda theory of cerebral lateralization, high intrauterine testosterone levels delay left brain hemisphere maturation and thus promote left handedness. Human circulating testosterone levels are higher in the male fetus and also vary with length of photoperiod. Therefore, a higher prevalence of left handedness, coupled with seasonal anisotropy (i.e., a non uniform distribution of handedness across birth months or seasons), may be expected among men. Prior studies yielded inconsistent evi dence for seasonal anisotropy and suffered from confounding and a number of shortcom ings affecting statistical power. This study examined hand preference and associations of handedness with sex, age, and season of birth in independent discovery $(n=7658)$ and replication $(n=5062)$ samples from Central Europe with latent class analysis (LCA). We found clear evidence of a surplus of left handed men born during the period November January, which is consistent with predictions from the Geschwind Galaburda theory. Moreover, seasonal anisotropy fully accounted for the higher prevalence of left handedness among men, relative to women. Implications of these findings with regard to seasonal anisotropy research and handedness assessment and classification are discussed.
\end{abstract}

\section{Introduction}

Limb preference and handedness are phenomena of many biological species. In humans, a dominant preference of the right limb for manual tasks is culturally universal (with a prevalence rate of right handedness of about $90 \%$; Coren, 1993; Peters, Reimers, \& Manning, 2006) and has been linked to brain lateralization. Whilst the genetic bases of handedness are still under debate (McManus, Davison, \& Armour, 2013; Medland et al., 2009), there have been numerous accounts investigating its associations with human capacities and traits.

However, research into handedness has also produced some fickle and elusive results. Whilst a higher prevalence of left handedness among men appears to be a robust finding

* Corresponding author. Department of Basic Psychological Research and Research Methods, School of Psychology, University of Vienna, Liebiggasse 5, A 1010 Vienna, Austria.

E mail address: ulrich.tran@univie.ac.at (U.S. Tran). 
(Papadatou Pastou, Martin, Munafò, \& Jones, 2008), the evi dence for some assumed biological underpinnings of left handedness appear inconclusive. The cerebral lateralization theory of Geschwind Galaburda (Geschwind \& Galaburda, 1985a, 1985b, 1985c, 1987) posits that high intrauterine levels of testosterone promote the development of left handedness because of differential effects of testosterone on brain hemi sphere maturation; specifically, left brain hemisphere matu ration is delayed. Male brains are exposed to substantially higher testosterone levels than female brains during prenatal development; hence, a sex effect for handedness may be ex pected. Furthermore, the secretion of sex hormones is higher during seasons with a long photoperiod, i.e., extended day length (Macchi \& Bruce, 2004). As a consequence, the theory also suggests a birth seasonality effect, i.e., seasonal anisot ropy, with regard to handedness.

There is direct (Lombardo et al., 2012) and indirect (Beaton, Rudling, Kissling, Taurines, \& Thorne, 2011) evidence for as sociations of intrauterine testosterone levels with brain development and left handedness in men. In contrast, evi dence for seasonal anisotropy is highly inconsistent: studies have reported anisotropy for widely different periods of the year, alternately affecting either men, or women, or both sexes likewise, or have found no effect at all (Stoyanov, Nikolova, \& Pashalieva, 2011). One meta analysis (Jones \& Martin, 2008), based on 48581 participants from 11 primary studies, has concluded that in the Northern hemisphere more left handed men are born during the period March-July. This meta analysis has been criticized with regard to the handed ness classification in the primary studies (Beaton, 2008). Indeed, its conclusion runs counter to expectation, because in the Northern hemisphere the photoperiod is the longest dur ing May-July. However, the critical period of testosterone induced human sexual differentiation does not occur perina tally, but much earlier prenatally, during gestational weeks 8-24; afterwards, plasma testosterone levels are generally low (Baron Cohen, Lutchmaya, \& Knickmeyer, 2004). The peak of embryonic neuronal migration also occurs during gestational week 12-20 (Tau \& Peterson, 2010). Testosterone induced ef fects on brain lateralization thus likely take place early during embryonic development. For the Northern hemisphere one would therefore expect more left handed men to be born during November-January, not during March-July, as sug gested by the meta analysis. Some studies indeed have re ported a higher prevalence of left handedness or non right handedness among men born in winter and/or autumn (Badian, 1983; Preti, Lai, Serra, \& Zurrida, 2008; Stoyanov et al., 2011). However, in light of the accumulating inconsistent findings in general, more evidence is clearly needed. More over, studies on this theme need to take into account specific validity threats and statistical power considerations, as elab orated below.

Whilst the effects of sex hormones and sex chromosomes on brain differentiation are likely more complex than previ ously thought (Lentini, Kasahara, Arver, \& Savic, 2012; Lust et al., 2011), available research in this area suffers from at least three different sources of bias and confounding which all threaten reliability and decrease statistical power. First, sample size is a pervasive problem in all handedness related research. The skewed 10:90 handedness ratio lowers the power of statistical tests by about $50 \%$, as compared to an optimally powered 50:50 ratio. Alternatively, oversampling left handers may result in dissimilarity of sampled pop ulations and thus introduce additional bias.

Second, handedness classification is often problematic. Hand preference in writing is molded by social and cultural pressure (Preti et al., 2011), which might lead to an underes timation of the true associations of handedness with other variables, when handedness classification is solely based on the hand used for writing (Papadatou Pastou et al., 2008). More reliable multi item inventories, like the Edinburgh Handed ness Inventory (EHI; Oldfield, 1971) or the Lateral Preference Inventory (LPI; Coren, 1993), still use arbitrarily adopted criteria and either a dichotomy (right/left, right/non right) or a trichotomy (right/mixed/left) for handedness classification. This may prohibit direct comparisons between studies and thus renders meta analytical results questionable (Beaton, 2008). Moreover, such varying classification criteria may even lead to different conclusions derived from the same data (Denny, 2008; Kelley, 2012). What is more, scoring and classi fication typically assigns equal weight to all items within an inventory. However, psychometrically this may be inappro priate (Dragovic, 2004). Existing taxometric (Dragovic, Milenkovic, \& Hammond, 2008) and latent class analyses (Büsch, Hagemann, \& Bender, 2009; Dragovic \& Hammond, 2007; see Methods) suggest that handedness in actual fact is discrete and thus support classification approaches. However, studies on handedness associations with other variables seldom make use of latent variable analyses that would allow for a differential weighting of indicators and the direct modeling of handedness.

Third, photoperiod in the Northern hemisphere is longest during May-July, shortest during November-January, and intermediate during February-April and August-October. Some studies have based their analyses either on the four meteorological seasons (winter: December-February; spring: March-May; summer: June-August; autumn: Septem ber-November; e.g., Stoyanov et al., 2011) or on the astro nomical seasons (winter: December 21/22-March 19/20; spring: March 20/21-June 20; summer: June 21-September 21/ 22; autumn: September 22/23-December 20/21; e.g., Tonetti, Adan, Caci, Fabbri, \& Natale, 2012). Other studies have con trasted half year periods (meteorological autumn and winter versus spring and summer; Jones \& Martin, 2008) or have used a monthly resolution for analysis (e.g., Milenković, Rock, Dragović, \& Janca, 2008). However, the meteorologically or astronomically defined seasons match with the seasons, as defined by photoperiod variation, by at most about two thirds, and monthly analyses neglect the non independence of consecutive months.

In the present study an attempt was made to overcome all of the above shortcomings. Given the inconsistency of past research, we investigated handedness and its seasonal anisotropy in a discovery sample as well as in an independent replication sample, in order to assess the replicability of our own initial findings. Independent discovery and replication samples within the same study are now considered best practice in genome wide association studies, in order to guard against false positive findings and to demonstrate the robustness of an effect, if the replication is successful 
(McCarthy et al., 2008). More generally, there is also an increased awareness of widespread replication failures and the irreproducibility of initially reported research findings across the medical and social sciences, which likewise has recently led to recommendations to intensify replication ef forts (Asendorpf et al., 2013). Recommendations for incentiv izing replication efforts (Button \& Munafò, 2014) and to reward high power replication research (Wagenmakers \& Forstmann, 2014) have also recently been published in this journal. Our study design thus conformed to these recent recommenda tions to counteract potentially false positive and thus irre producible research findings.

We utilized latent class analysis (LCA) for the classification of handedness and examined the associations of handedness with sex, age, and season of birth. Specifically, we expected a surplus, i.e., seasonal anisotropy, of left handed men born during the period November-January.

\section{Methods}

\subsection{Participants}

In designing the study, independent discovery and replication samples were implemented. Descriptive statistics for the two samples are given in Table 1. Overall, both samples in the majority comprised Austrian and German participants and slightly more women than men. Participants from other countries were almost exclusively Caucasians; the further nationalities with the highest proportions were Italian (1.4\%), Turkish (.6\%), and Romanian (.4\%). Range of participant age was broad.

\subsection{Procedure and measures}

The data were collected by a multitude of data collectors in the course of larger projects on individual differences variables (see Tran, Koller, et al., 2014, Tran, Stieger, \& Voracek, 2014). The two data collection waves, yielding the discovery and the replication sample, were temporally separated by about one half year and independent from each other regarding the data collectors involved. The data collectors were geographi cally diverse and, mutually independent from each other, collected the data for course credit. In addition to basic de mographic information and a number of further individual

Table 1 - Sample descriptive statistics.

\begin{tabular}{lll}
\hline & Discovery sample & Replication sample \\
\hline$n$ & 7658 & 5062 \\
Women, $n(\%)$ & $4456(58.2 \%)$ & $2749(54.3 \%)$ \\
Age, range (years) & 1889 & 1892 \\
$\quad$ Interquartile range & 2235 & 2248 \\
$\quad$ Mean (SD) & $30.24(12.62)$ & $35.74(16.05)$ \\
Nationality, $n(\%)^{\mathrm{a}}$ & & \\
$\quad$ Austria & $5339(70.0 \%)$ & $3415(67.7 \%)$ \\
$\quad$ Germany & $1773(23.2 \%)$ & $1310(26.0 \%)$ \\
$\quad$ Other & $514(6.7 \%)$ & $316(6.3 \%)$ \\
\hline a & ns $=7626$ and 5041 because of partially missing data. \\
\hline
\end{tabular}

differences measures, all participants provided hand prefer ence ratings according to the German translation of four items selected from the LPI (Coren, 1993). Participants were approached on a personal basis, utilizing personal contacts and word of mouth advertising. Participants had to be fluent in German, as this was the survey language. Apart from insufficient language proficiency, there were no further exclusion criteria. Study participation was voluntary, anony mous, and participants were not remunerated for participation.

For survey economy, four items from of the full version, 12 item LPI handedness scale were selected for this research: hand preference with regard to writing (Item 1), throwing a ball to hit a target (throwing; Item 3), using a knife to cut something without simultaneously using a fork (knife; Item 6), and using a hammer to drive a nail into something (hammer, Item 7). Response options were left, either, and right (in this order and reading direction). For handedness classifi cation, the consistency of the preferences across this item set may be utilized (thereby distinguishing consistent from inconsistent right handers), or, alternatively, a total score may be computed, with a coding of left 1 , either 0 , and right +1 applied, and then summing up across all items. The sign and magnitude of the total score across the items is then indicative of the direction and strength of hand preference, respectively. Coren (1998) reported that self reported hand preferences according to the LPI were $96 \%$ concordant with respondents' behavioral performance on the same motor ac tions. As mentioned above, handedness was assessed in addition to further variables for a larger individual differences project. For the sake of efficiency, this assessment was thus deliberately kept brief. Apart from assessing the adult writing hand (Item 1), items were chosen with respect to broad trait coverage (us redundancy), balance of fine motor versus gross motor skills represented in the specific item contents, and beneficial item performance indicators in particular. The items probing the writing hand, the throwing hand, and holding a knife had excellent psychometric properties (i.e., high squared multiple correlations of items with test scores) in a recent psychometric study of Dragovic (2004) as well as in a replication study of Milenkovic and Dragovic (2013), who developed a refined version of the 10 item EHI (Oldfield, 1971). The EHI shares six items with the LPI, and the refined 7 item version of Milenkovic and Dragovic (2013) includes three of the items (writing hand, throwing hand, and holding a knife) selected for the present study. Sample reliability figures (Cronbach $\alpha$ ) for the full 12 item LPI frequently approaches .95 (e.g., Voracek, Reimer, Ertl, \& Dressler, 2006). In the present study, Cronbach $\alpha$ values for the abbreviated 4 item scale were .91 and .92 in the discovery and replication samples, respectively, and .91 , when both samples were combined. That is, reliability of the 4 item selection administered here was nearly as high as the reliability typically observed for the complete (12 item) LPI.

\subsection{Analysis}

LCA was used to investigate the preference ratings in the LPI. LCA is based on a latent variable model, wherein associations between observed variables (indicators) are explained by a 
Table 2 - Frequencies of hand preference across the administered LPI items.

\begin{tabular}{|c|c|c|c|c|c|c|}
\hline \multirow[t]{2}{*}{ Item } & \multirow[t]{2}{*}{ Sample } & \multicolumn{3}{|c|}{ Hand preference } & \multirow[t]{2}{*}{ Missing values } & \multirow[t]{2}{*}{$\chi^{2}$} \\
\hline & & Right & Left & Either & & \\
\hline \multirow[t]{2}{*}{ Writing } & Discovery & 7006 (91.6\%) & $582(7.6 \%)$ & $60(.8 \%)$ & 10 & .17 \\
\hline & Replication & 4624 (91.4\%) & 394 (7.8\%) & $41(.8 \%)$ & 3 & \\
\hline \multirow[t]{2}{*}{ Throwing } & Discovery & $6836(89.4 \%)$ & $553(7.2 \%)$ & $257(3.4 \%)$ & 12 & $8.41^{*}$ \\
\hline & Replication & 4501 (89.1\%) & $414(8.2 \%)$ & $135(2.7 \%)$ & 12 & \\
\hline \multirow[t]{2}{*}{ Knife } & Discovery & $6805(89.0 \%)$ & 604 (7.9\%) & 239 (3.1\%) & 10 & $9.62^{* *}$ \\
\hline & Replication & $4525(89.4 \%)$ & 423 (8.4\%) & $113(2.2 \%)$ & 1 & \\
\hline \multirow[t]{2}{*}{ Hammer } & Discovery & $6853(89.6 \%)$ & 581 (7.6\%) & $214(2.8 \%)$ & 10 & 3.77 \\
\hline & Replication & $4514(89.3 \%)$ & 419 (8.3\%) & $121(2.4 \%)$ & 8 & \\
\hline
\end{tabular}

Note. $\chi^{2}=\chi^{2}$ test of independence, $d f=2$. Note that the statistical tests were overpowered, owing to the large samples involved; observed between sample differences are negligible. ${ }^{*} p<.05,{ }^{*} p<.01$.

number of discrete latent classes (Collins \& Lanza, 2010). Formally, the statistical model of LCA may be expressed as a subtype of structural equation modeling and therefore shares many of its advantageous features. Specifically, LCA allows for a differential weighting of indicators with regard to class assignment (just like in factor analysis with regard to the estimation of the continuous latent trait).

We evaluated the fit of a number of latent class models, determining empirically the smallest number of latent classes that optimally accounted for the data observed in the dis covery and the replication sample. In these analyses, items were treated as nominal, because of the evidence for a discrete nature of handedness (Büsch et al., 2009; Dragovic \& Hammond, 2007; Dragovic et al., 2008). Model fit was assessed with the log likelihood value (LL) and the Bayesian information criterion, BIC(LL), which is based on the LL. Higher LL and lower BIC(LL) values indicate better model fit. Furthermore, we applied the likelihood ratio goodness of fit statistic $\left(L^{2}\right)$ to compare the observed item response fre quencies with the expected frequencies, as predicted by the model, and calculated the percentages of classification error. Lower (and preferably statistically not significant) $L^{2}$ values and lower percentages of classification error indicate better model fit. LCA was conducted with Latent GOLD 4.5 (Vermunt \& Magidson, 2005). Sixty four (.5\%) participants had 1 missing value and one participant 2 missing values for the four LPI items, which cases were included in the analysis. The latent handedness classes identified were used in the subsequent seasonal anisotropy analyses of handedness.

The hand preference observed in the LPI was investigated with $\chi^{2}$ tests of independence, and the associations of hand edness with sex, age, and season of birth with logistic regression analyses and analyses of variance (ANOVA). Replicability across samples was assessed in combined samples analyses by adding a main effect and interactions with regard to type of sample (discovery us replication) in lo gistic regression models and analyses of variance. In these tests, significant interaction effects would indicate non replicability of findings, i.e., effects not emerging in both the discovery and the replication sample. In the tests for birth month distribution and seasonality of handedness, we applied corrections for baseline differences regarding the total number of births per month in the samples; that is, the observed frequencies were differentially weighted to account for these baseline differences. This adjustment also corrected for baseline differences due to the unequal length of months.

\section{Results}

\subsection{Sample differences in demographics}

The discovery sample comprised about $4 \%$ more women than the replication sample $\left[\chi^{2}(1) \quad 18.69, p<.001\right]$. Participants in the replication sample were on average 5.5 years older $[t(12718) \quad 21.56, p<.001, d \quad .39]$. Owing to the large samples, these inferential statistical tests clearly were overpowered and thus both nominally significant, albeit these differences would seem practically negligible.

\subsection{Hand preference in the LPI}

Table 2 lists the observed response frequencies on the four handedness items from the LPI. Endorsement of response categories were similar in the discovery and the replication sample. Between sample differences, wherever these were nominally significant, overall were negligible $(\leq 1 \%)$. Right hand preference ranged from $89.0 \%$ to $91.6 \%$ across samples and items, left hand preference from $7.2 \%$ to $8.4 \%$. The prev alence of mixed handed writing was low $(.8 \%)$, whereas a preference for both hands was reported by $2.2 \%-3.4 \%$ for the other three LPI items.

\subsection{Latent structure of handedness}

Table 3 displays the fit of $1,2,3$, and 4 cluster models in the discovery and the replication sample. According to the BIC(LL) and the percentages of classification error, in both samples a 3 cluster solution fitted the data best. The mean posterior assignment probabilities of participants to clusters were high for Class 1 (99.4\% and $99.4 \%$ in the discovery and the replica tion sample, respectively) and Class 2 (96.9\% and 96.9\%), whereas somewhat lower for Class 3 ( $89.4 \%$ and $90.0 \%)$, but overall indicating a high classification certainty. Fig. 1 depicts the estimated response probabilities for the 3 cluster model in the discovery and the replication sample.

Class 1 , subsuming right handed individuals, had class sizes of $89.5 \%$, with a $95 \%$ confidence interval of [ $88.7-90.3 \%$ ] 
Table 3 - Fit of latent class models for handedness classification.

\begin{tabular}{lrrrrrrr}
\hline Model & LL & Npar & BIC(LL) & $L^{2}$ & $d f$ & $p$ & Classification error, \% \\
\hline Discovery sample & & & & & & & \\
1 cluster & 11665.52 & 8 & 23402.59 & 8441.22 & 184 & $<.001$ & .00 \\
2 cluster & 7908.81 & 17 & 15696.66 & 927.79 & 175 & $<.001$ & .52 \\
3 cluster & 7551.28 & 26 & 15335.10 & 212.74 & 166 & .008 & 1.03 \\
4 cluster & 7511.84 & 35 & 15336.69 & 133.84 & 157 & .910 & 3.82 \\
Replication sample & & & & & & \\
1 cluster & 7652.41 & 8 & 15373.05 & 5871.32 & 176 & $<.001$ & .00 \\
2 cluster & 4988.65 & 17 & 10122.31 & 543.80 & 167 & $<.001$ & .49 \\
3 cluster & 4805.94 & 26 & 9833.65 & 178.38 & 158 & .130 & .05 \\
4 cluster & 4782.74 & 35 & 9864.01 & 131.98 & 149 & .840 & 3.58 \\
\hline
\end{tabular}

Note. $L=$ log likelihood; Npar = number of parameters; $B I C(L L)=$ Bayes information criterion based on LL; $L^{2}=$ likelihood ratio test statistic.

in the discovery sample and of $89.0 \%[88.0-90.0 \%]$ in the replication sample. Consistency of hand preference was high: response probabilities of right hand preference were $\geq 97 \%$ on any item, and $\leq 2 \%$ for mixed hand and left hand preference.

Class 2, subsuming left handed individuals, had class sizes of $7.7 \%$ [7.1-8.3\%] and $8.4 \%$ [7.6-9.3\%], respectively. Consis tency of hand preference in Class 2 was lower than in Class 1 : response probabilities of left hand preference were only $\geq 74 \%$, with the lowest values for the writing hand item ( $80 \%$ in the discovery and $74 \%$ in the replication sample, respectively), and the highest values for the hammer item ( $91 \%$ and $92 \%)$. In return, response probabilities of right hand preference were highest for writing, $19 \%$ and $25 \%$ in the discovery and the replication samples, but lowest for hammer, $6 \%$ and $6 \%$, respectively. The higher probability of right handed writing in the replication sample did not differ significantly from the discovery sample, as the respective $95 \%$ confidence intervals overlapped. Comparable to Class 1 , mixed hand response probabilities were $\leq 4 \%$ on any item in Class 2 .

Class 3 had a class size of $2.8 \%[2.3-3.4 \%]$ and $2.6 \%$ [1.9-3.3\%], respectively, and subsumed mixed handed
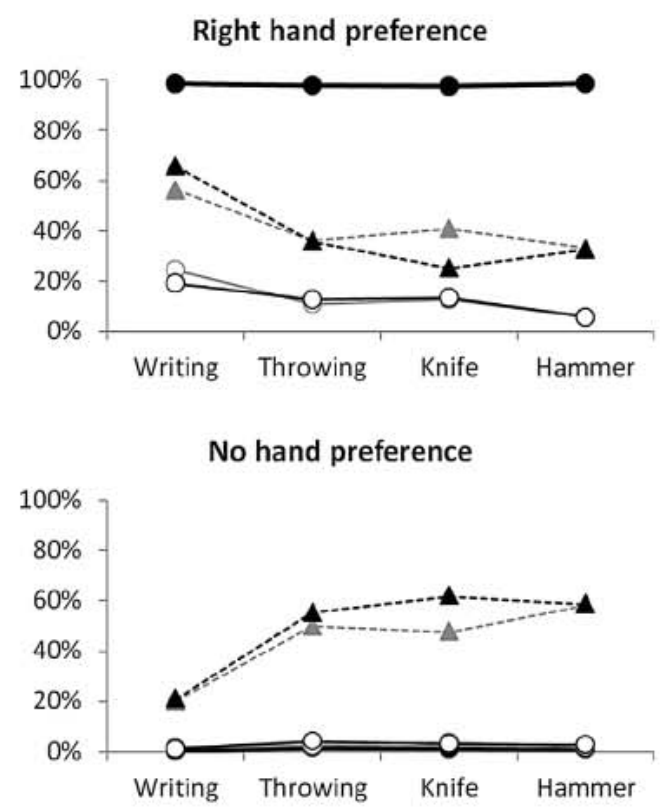

participants. Members of Class 3 had response probabilities of using either hand in throwing, knife, and hammer of $\geq 48 \%$, which consistently were highest across both samples for the hammer item, $59 \%$ and $58 \%$ in the discovery and the replica tion sample, respectively. Mixed handed writing had response probabilities of $21 \%$ and $20 \%$. Otherwise, members of Class 3 preferred the right over the left hand: right handed writing had response probabilities of $66 \%$ and $56 \%$, as compared to $13 \%$ and $24 \%$ for left handed writing. With the exception of the knife item ( $25 \%$ in the discovery sample), across both samples right hand preference had response probabilities of at least $33 \%$ on all other items, as compared to a maximum of $24 \%$ (throwing) with regard to left hand pref erence. None of the response probabilities differed signifi cantly between the samples in Class 3 (all respective 95\% confidence intervals overlapped).

Overall, Item 4 (hammer) of the LPI contributed strongest in differentiating the latent classes. In the 3 cluster model, the loading of Item 4 was .85 and .87 in the discovery and the replication sample, respectively, whereas loadings of the other three items ranged between .74 and .81 in the two

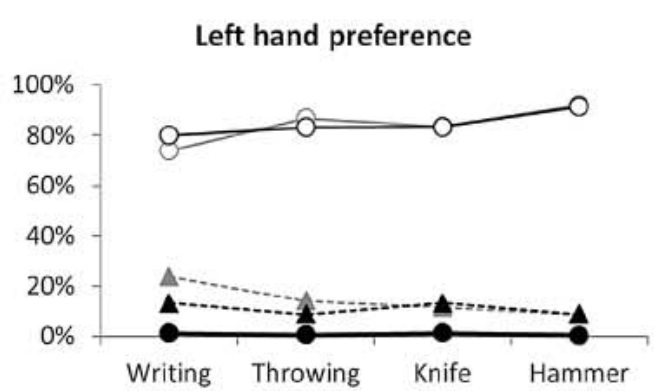

$\rightarrow$ Class 1

$\sim$-Class 2

--A- Class 3

Fig. 1 - Estimated response probabilities in the 3-cluster model. Black lines depict probabilities in the discovery sample, gray lines the respective probabilities in the replication sample. 
Table 4 - Distribution of class membership within participant sex.

\begin{tabular}{llcccc}
\hline Sample & & Class 1 & Class 2 & Class 3 & $\chi^{2}$ \\
\hline Discovery & Men & $2854(89.1 \%)$ & $260(8.1 \%)$ & $88(2.7 \%)$ & 2.69 \\
& Women & $4019(90.2 \%)$ & $335(7.5 \%)$ & $102(2.3 \%)$ & $48(2.1 \%)$ \\
Replication & Men & $2038(88.1 \%)$ & $227(9.8 \%)$ & $59(2.1 \%)$ & $9.24^{* *}$ \\
& Women & $2486(90.4 \%)$ & $204(7.4 \%)$ & & \\
Note. $\chi^{2}=\chi^{2}$ test of independence, $d f=2 . " p<.01$. & & &
\end{tabular}

samples. Accordingly, prediction of class membership through Item 4 yielded the highest classification accuracy of all items: across both samples, the positive predictive value of Item 4 (hammer) was $98.8 \%$ for Class 1 (right handedness), $92.9 \%$ for Class 2 (left handedness), and $60.0 \%$ for Class 3 (mixed handedness).

Also, class membership reliably discriminated participants with regard to their LPI scores. Class membership explained $91 \%$ of total score variability in the two samples [ANOVA with factors class and sample; class: $F(2,12649) \quad 64070.11, p<.001$, $\eta_{p}^{2} \quad .910$; sample: $F(1,12649) \quad 2.52, p \quad .112, \eta_{p}^{2}<.001$; inter action: $\left.F(2,12649) \quad 4.38, p \quad .013, \eta_{p}^{2} \quad .001\right]$, with members of Class 1 (right handers) scoring highest ( $M \quad 3.90, S D \quad .40$ ),

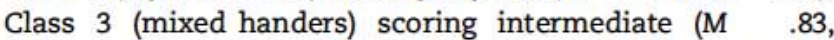
$S D$ 1.15), and Class 2 (left handers) scoring lowest (M 2.84, SD 1.43). Given the fact that the internal con sistency (Cronbach $\alpha$ ) of the four LPI items was .91, it follows that essentially all reliable LPI score variance was explained by class membership (as indicated by the above effect size: $\eta_{p}^{2} \quad$.910). Members of Class 1 also scored somewhat lower in the discovery than in the replication sample (significant interaction); however, this difference was practically negli gible $\left(\eta_{p}^{2}<.001\right)$.

\subsection{Associations of handedness with sex, age, and season of birth}

The distribution of class membership with regard to partici pant sex is presented in Table 4. In both samples, the pro portion of Class 2 members (left handers) was slightly elevated among men, relative to women. This effect was sig nificant in the replication sample, but not in the discovery sample (see Table 4). In the logistic regression analysis, no sex effect was obtained overall; however, the test for non replicability (interaction effect of sex with sample) also failed to reach nominal significance ( $p \quad .094$; see Table 5 , column 1 ). When this interaction term was omitted from the model, the sex effect became significant (OR 1.19 [1.05-1.36], $p \quad .007$ ). We accepted this result as suggestive of an overall sex dif ference in Class 2 proportions that amounted to a $1.3 \%$ [.4-2.3\%] difference (overall proportions of Class 2 were $8.8 \%$ among men and $7.5 \%$ among women).

No association of class membership with age could be observed [ANOVA with factors class and sample; class: $F(2$, 12714) $.61, p \quad .545, \eta_{p}^{2}<.001$; sample: $F(1,12714) \quad 72.73$, $p<.001, \eta_{p}^{2} \quad .006$; interaction: $F(2,12714) \quad .01, p \quad .993$, $\eta_{p}^{2} \quad .001$ ]. However, older age among members of Class 2 (left handers) specifically predicted right handed writing (see Table 5, column 2). Members of Class 2 had their odds raised by a factor of 3.12 [2.55-3.81] per 10 years increase of age to report right hand writing. No further association of hand preference with age was observed for any of the other three LPI items in Class 2 ( $p s \geq .152$ for the overall models; results omitted for brevity).

The birth month distribution of class membership is depicted in Fig. 2, collapsing the discovery and the replication samples and omitting Class 3 (mixed handers) for which at most 20 observations were available for each month. Conse quently, the respective estimates of the proportions were deemed unreliable and therefore not used in Fig. 2. Among men, the monthly proportions of Class 2 (left handers) exceeded that of Class 1 (right handers) consecutively from November to January by $1.4 \%-1.9 \%$, whereas no such pattern was observable for women. Compared to the remainder of the year, among men (but not women) the proportion of left handedness was significantly higher during the period November-January (see Table 5, columns 3 and 4). Overall, the difference in Class 2 proportions among men with regard to the period November-January (10.5\%) and the remainder of the year $(8.2 \%)$ amounted to $2.3 \%$ [.4-4.1\%]. Moreover, sea sonality fully accounted for the difference in the proportions of left handedness between men and women (see Table 5, column 5). Of all female Class 2 members, $21.5 \%$ were born during November-January, whereas among men the corre sponding figure was $29.9 \%$.

In seasonality analyses (Table 5 , columns $3-5$ ), left handedness was contrasted with the mixed handedness and right handedness groups combined. In additionally calculated analyses, combined groups of left handedness and mixed handedness were contrasted with the right handedness group. This alternative analysis however left the above find ings essentially intact (details omitted for brevity). In partic ular, the sex by season interaction remained significant and was of comparable magnitude as in the above analysis, OR 1.45 [1.08-1.95], $p \quad .014$. In similar vein, conducting the seasonality analyses separately for the two samples did not yield results contradicting those of the above analysis. The sex by season interaction was clearly significant in the repli cation sample, OR 1.97 [1.22-3.19], $p \quad .006$, and only closely missed nominal significance in the discovery sample, OR 1.46 [.99-2.14], $p \quad .054$, which may be attributed to the relative loss of statistical power in these separate sample analyses, as compared to the combined sample analysis. Of note, confidence intervals of these sex by season effect esti mates from the separate sample analyses overlapped considerably, thus suggesting comparable effects. Further more, in the separate sample analyses there also emerged a main effect of season in the replication sample, OR .65 [.45-.95], $p \quad .025$, but not in the discovery sample, OR $\quad .91$ [.70-1.19], $p \quad .499$. However, in the presence of the significant 
Table 5 - Associations of left-handedness (Class 2) with sex, age, and season of birth according to logistic regression analyses.

\begin{tabular}{|c|c|c|c|c|c|}
\hline & \multirow{2}{*}{$\begin{array}{c}\text { Sex: Class } 2 \\
\text { versus classes } \\
1 \text { and } 3 \\
\end{array}$} & \multirow{2}{*}{$\begin{array}{l}\text { Age: Right handed } \\
\text { writing among } \\
\text { members of Class } 2^{\text {a }}\end{array}$} & \multicolumn{3}{|c|}{ Season of birth: Class 2 versus classes 1 and $3^{b}$} \\
\hline & & & Men & Women & Sexes combined \\
\hline Sex ${ }^{c}$ & $1.09\left[\begin{array}{ll}.92 & 1.29\end{array}\right]$ & & & & $1.05\left[\begin{array}{ll}.91 & 1.22\end{array}\right]$ \\
\hline Age & & $1.12\left[\begin{array}{ll}1.10 & 1.14\end{array}\right]^{* * *}$ & & & \\
\hline Season ${ }^{d}$ & & & $1.33\left[\begin{array}{ll}1.01 & 1.76\end{array}\right]^{*}$ & $.91\left[\begin{array}{ll}.70 & 1.19\end{array}\right]$ & $.81\left[\begin{array}{ll}.66 & 1.01\end{array}\right]$ \\
\hline Sample & $.99\left[\begin{array}{ll}.82 & 1.18\end{array}\right]$ & $.58\left[\begin{array}{ll}.16 & 2.12\end{array}\right]$ & $1.27\left[\begin{array}{ll}1.01 & 1.58\end{array}\right]^{*}$ & $1.06\left[\begin{array}{ll}.86 & 1.29\end{array}\right]$ & $1.09\left[\begin{array}{ll}.94 & 1.25\end{array}\right]$ \\
\hline Sex $\times$ season & & & & & $1.53\left[\begin{array}{ll}1.09 & 2.15\end{array}\right]^{*}$ \\
\hline \multicolumn{6}{|l|}{ Tests of nonreplicability } \\
\hline Sex $\times$ sample & $1.25\left[\begin{array}{ll}.96 & 1.62\end{array}\right]$ & & & & \\
\hline Age $\times$ sample & & $1.00\left[\begin{array}{ll}.97 & 1.04\end{array}\right]$ & & & \\
\hline Season $\times$ sample & & & $.96\left[\begin{array}{ll}.64 & 1.46\end{array}\right]$ & $.72\left[\begin{array}{ll}.45 & 1.13\end{array}\right]$ & \\
\hline Sex $\times$ season $\times$ sample & & & & & $1.13\left[\begin{array}{ll}.78 & 1.64\end{array}\right]$ \\
\hline Model fit $\chi^{2}(d f)$ & $12.40(3)^{* *}$ & $371.86(3)^{*+1}$ & $12.01(3)^{* *}$ & $5.90(3)$ & $20.02(5)^{* *}$ \\
\hline Nagelkerke $R^{2}$ & .002 & .478 & .005 & .002 & .004 \\
\hline \multicolumn{6}{|c|}{$\begin{array}{l}\text { Note. Cells entries are odds ratios with } 95 \% \text { confidence intervals. }{ }^{*} p<.05, "{ }^{*} p<.01, " * p<.001 . \\
\text { a } n=1013 \text { of whom } 213 \text { reported right handed writing. } \\
\text { b Corrected for baseline differences in numbers of births per month. Coding: } \\
\text { c } 1=\text { men, } 0=\text { women. } \\
\text { d } 1=\text { November January, } 0=\text { remainder of the year. } \\
\text { e } 1=\text { replication sample, } 0=\text { discovery sample. }\end{array}$} \\
\hline
\end{tabular}

sex by season interaction (see above), the significant main effect of season in the replication sample simply mirrored the relatively stronger overall effect of sex on handedness in this sample (see above).

Notably, comparing the meteorological winter with the remainder of the year, a seasonality effect could not be veri fied (logistic regression model as in Table 5, column 5; sex by season interaction: $\mathrm{OR} \quad 1.27$ [.90-1.79], $p \quad .183$; further de tails omitted for brevity). Likewise, no association of season of birth with Class 2 membership was observed by comparing the half year periods August-February (autumn and winter) and March-July (spring and summer; sex by season interac tion: OR $1.06[.79-1.42], p \quad .691$; further details omitted for brevity).

\section{Discussion}

This study set out to reassess predictions for seasonality of handedness, following the theory of Geschwind Galaburda, using two large and independent samples and LCA of self reported handedness data. Our results suggest that, first, three classes of handedness need to be considered - right handedness, left handedness, and mixed handedness. Sec ond, handedness appeared to be independent of age, but not of sex and season of birth: men were more often left handed than women, and more left handed men were born during the period November-January than during the remainder of the year, which consorts well with predictions from the Geschwind-Galaburda theory. Third, in the present data this
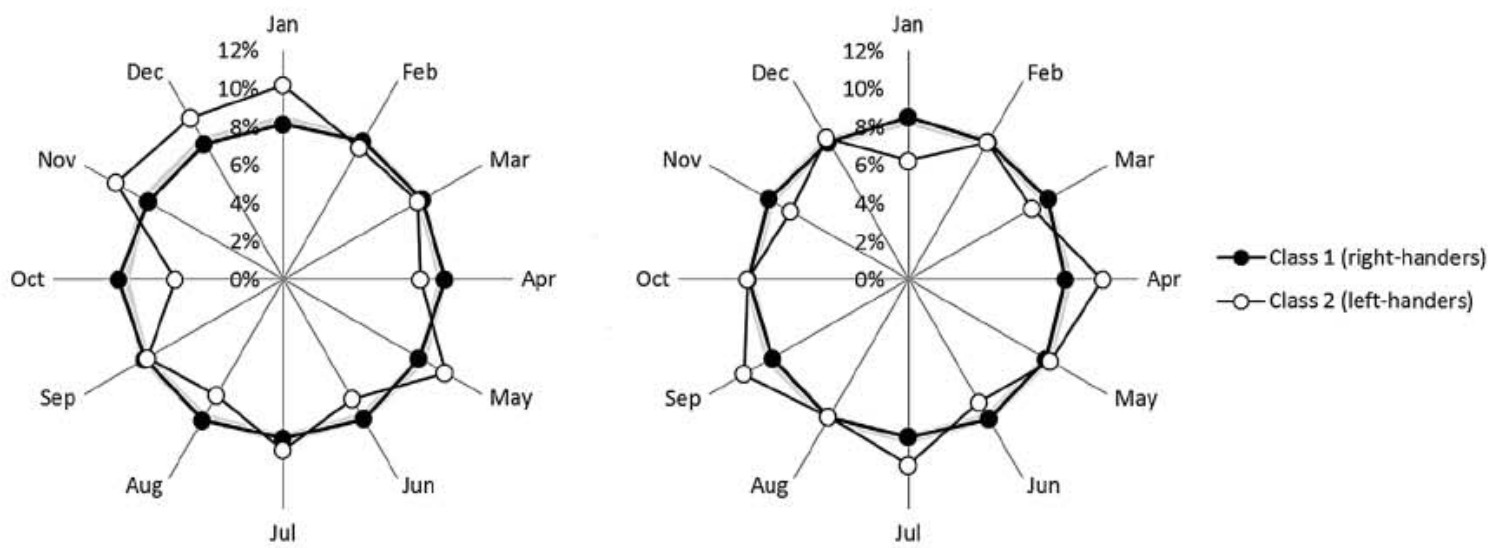

Fig. 2 - Radial plots of the birth month distribution of class membership among men (left) and women (right), comparing the Classes 1 and 2, with the discovery and replication samples combined. Proportions are corrected for baseline differences in numbers of births per month. Gray lines signify the constant proportion of $8.3 \%$ that would be expected for each handedness class under the assumption of uniform distribution of handedness across birth months. 
seasonal anisotropy fully explained the higher prevalence of left handedness among in men, relative to women.

Thus, even though previous results on seasonal anisotropy were inconsistent, this study provides clear evidence in sup port of one prediction derived from the theory of Gesch wind-Galaburda and therefore adds to the prior positive evidence of Badian (1983), Preti et al. (2008), and Stoyanov et al. (2011). Furthermore, because according to our data seasonal anisotropy specifically affected men born during the period November-January, as expected, one may infer that the crit ical period of testosterone induced effects on brain laterali zation effectively is limited to gestational weeks 14-18. Otherwise, individuals born in both the precedent and sub sequent months should have been affected to some extent as well. Seasonal anisotropy was specifically confined to men, as in the reports of Badian (1983) and Stoyanov et al. (2011).

The proposed short critical period corresponds to the magnitude of the effects observed in this study: men's preva lence rate of left handedness exceeded women's by $1.3 \%$ (which broadly corresponds to previous estimates; Peters et al., 2006), and among men the overall prevalence of left handedness of birth cohorts of November-January exceeded that of the remainder of the year by only $2.3 \%$. In contrast, the difference in the proportions of left handedness among men and women for the period November-January, $29.9 \%$ us $21.5 \%$, appears sizable. Nonetheless, given the overall low prevalence of left handedness, large samples and high precision of handedness classification are mandatory to spot an effect of this size. For these combined reasons, previous studies could not provide a clear picture on this seasonal anisotropy effect. In support of these arguments is the fact that we were unable to obtain the seasonality effect with our own data, when we, by way of trial, analyzed half year periods (Jones \& Martin, 2008) or seasons not aligned to photoperiod variation (Tonetti et al., 2012).

Even though in our data seasonal anisotropy fully accounted for the sex difference in left handedness, there conceivably are a number of further prenatal, perinatal, and postnatal candidate factors to consider for refined explana tion, such as maternal age, birth order, birth stress, or cultural factors (Badian, 1983; Bailey \& McKeever, 2004; Papadatou Pastou et al., 2008). Also, regional effects and latitude need to be taken into account vis à vis seasonal variation (Badian, 1983). For example, the null finding of the large ( $N$ 15390) study of Cosenza and Mingoti (1995) may have been caused by the only small seasonal variation in photoperiod at their study locale, which was situated close to the equator (Minas Gerais in Brazil; latitude $19.54^{\circ} \mathrm{S}$ ). In contrast, sampling in the pre sent study primarily took place in Central Europe (Austria and Germany), with the latitudes for these two countries extend ing from $46^{\circ}$ to $55^{\circ} \mathrm{N}$. We suggest that the seasonal variation in photoperiod - not merely meteorologically or astronomically defined seasons - together with the moderating effect of latitude on the extent of this seasonal variation might be beneficially taken into account in future research.

Regarding handedness assessment, our study provided prevalence estimates of right handedness (about 89\%) that are in line with many previous results. Our data are further indicative of a trichotomy of handedness, comprising right handedness, left handedness, and mixed handedness, which is consistent with recent taxonometric and latent class analyses (Dragovic \& Hammond, 2007; Dragovic et al., 2008). Overall, the prevalence of left handedness was about $8 \%$, whereas those of mixed handedness less than $3 \%$.

Analysis revealed that consistency of handedness was highest among right handers, but less so among non right handers. Inconsistent use of the left hand was more or less the norm among left handers, who frequently stated to prefer their right hand as well. Concerning the writing hand, we found strong evidence for a decreasing social and cultural pressure against left handedness among the more recent birth cohorts: older, but not younger, left handers frequently reported writing with their right hand (McManus, Moore, Freegard, \& Rawles, 2010). For this reason, hand preference for writing appears to be a relatively weak and moreover confounded indicator of handedness (Preti et al., 2011), which therefore should probably omitted from handedness classification.

Even though the group of mixed handers was only small and right hand preference was also frequent among these individuals, this was the only group which consistently and most often indicated no hand preference regarding the four manual tasks of the LPI. There have been accounts of mixed handedness alternately being described as detrimental (Crow, Crow, Done, \& Leask, 1998; Kelley, 2012; Nettle, 2003; Peters et al., 2006) or beneficial (Christman \& Propper, 2001; Denny, 2008; Niebauer, 2004) for various psychological abilities and health conditions. Our study suggests that inconsistent re sults such as these might partly stem from mingling mixed handers, who are characterized by no clear hand preference, with left handers, who are generally characterized by an inconsistent preference of their left hand. Clearly, more research is needed to shed more light on this specific topic.

Furthermore, we found evidence that a bimanual task which involves the risk of hurting oneself, if executed faultily (using a hammer to drive a nail into something), had the highest reliability of classifying handedness on its own. Bimanual tasks are assumed to confer a higher classification reliability (Papadatou Pastou et al., 2008). However, our study suggests that it may need to be a task which requires accuracy and, if executed faultily, exerts a direct negative or hurtful consequence to the individual performing it. This idea ought to be followed up, as it might improve the assessment and clas sification of handedness from inventories of hand preference.

Summing up, utilizing a discovery and replication sample approach, our study provided clear evidence for a seasonal anisotropy of left handedness among men, which effect is consistent with predictions derived from the Gesch wind-Galaburda theory. Future research along these lines may need to take more closely into account the validity threats and statistical power issues elaborated on here. Like wise, the moderating effects of latitude on the amount of seasonal variation of photoperiod should be further investi gated vis à vis seasonal anisotropy.

\section{Acknowledgments}

Thanks are extended to Ingrid Koller, Ingo W. Nader, Jakob Pietschnig, Anne H. E. Schild, and Elisabeth L. Zeilinger for supporting the data collection. 


\section{R E F E R E N C E S}

Asendorpf, J. B., Conner, M., De Fruyt, F., De Houwer, J., Denissen, J. J., Fiedler, K., et al. (2013). Recommendations for increasing replicability in psychology. European Journal of Personality, 27(2), 108119.

Badian, N. A. (1983). Birth order, maternal age, season of birth, and handedness. Cortex, 19(4), 451463.

Bailey, L. M., \& McKeever, W. F. (2004). A large scale study of handedness and pregnancy/birth risk events: implications for genetic theories of handedness. Laterality, 9(2), 175188.

Baron Cohen, S., Lutchmaya, S., \& Knickmeyer, R. C. (2004). Prenatal testosterone in mind: Amniotic fluid studies. Cambridge, MA: MIT Press.

Beaton, A. A. (2008). Handedness assessment in studies of seasonal anisotropy. Cortex, 44(1), 9798.

Beaton, A. A., Rudling, N., Kissling, C., Taurines, R., \& Thorne, J. (2011). Digit ratio (2D:4D), salivary testosterone, and handedness. Laterality, 16(2), 136155.

Büsch, D., Hagemann, N., \& Bender, N. (2009). The lateral preference inventory: the item homogeneity of the German version. Zeitschrift für Sportpsychologie, 16(1), 1728.

Button, K. S., \& Munafò, M. R. (2014). Incentivising reproducible research. Cortex, 51(February), 107108.

Christman, S. D., \& Propper, R. E. (2001). Superior episodic memory is associated with interhemispheric processing. Neuropsychology, 15(4), 607616.

Collins, L. M., \& Lanza, S. T. (2010). Latent class and latent transition analysis for the social, behavioral, and health sciences. New York: Wiley.

Coren, S. (1993). The left hander syndrome: The causes and consequences of left handedness. New York: Vintage Books.

Coren, S. (1998). Prenatal testosterone exposure, left handedness, and high school delinquency. Behavioral and Brain Sciences, 21(3), 369370.

Cosenza, R. M., \& Mingoti, S. A. (1995). Season of birth and handedness revisited. Perceptual and Motor Skills, 81(2), 475480 .

Crow, T. J., Crow, L. R., Done, D. J., \& Leask, S. (1998). Relative hand skill predicts academic ability: global deficits at the point of hemispheric indecision. Neuropsychologia, 36(12), 12751281.

Denny, K. (2008). Cognitive ability and continuous measures of relative hand skill: a note. Neuropsychologia, 46(7), 20912094.

Dragovic, M. (2004). Towards an improved measure of the Edinburgh handedness inventory: a one factor congeneric measurement model using confirmatory factor analysis. Laterality, 9(4), 411419.

Dragovic, M., \& Hammond, G. (2007). A classification of handedness using the Annett Hand Preference Questionnaire. British Journal of Psychology, 98(3), 375387.

Dragovic, M., Milenkovic, S., \& Hammond, G. (2008). The distribution of hand preference is discrete: a taxometric examination. British Journal of Psychology, 99(4), 445459.

Geschwind, N., \& Galaburda, A. M. (1985a). Cerebral lateralization. Biological mechanisms, associations, and pathology: I. A hypothesis and a program for research. Archives of Neurology, 42(5), 428459.

Geschwind, N., \& Galaburda, A. M. (1985b). Cerebral lateralization. Biological mechanisms, associations, and pathology: II. A hypothesis and a program for research. Archives of Neurology, 42(6), 521552.

Geschwind, N., \& Galaburda, A. M. (1985c). Cerebral lateralization. Biological mechanisms, associations, and pathology: III. A hypothesis and a program for research. Archives of Neurology, 42(7), 634654.
Geschwind, N., \& Galaburda, A. M. (1987). Cerebral lateralization: Biological mechanisms, associations and pathology. Cambridge, MA: MIT Press.

Jones, G. V., \& Martin, M. (2008). Seasonal anisotropy in handedness. Cortex, 44(1), 812.

Kelley, M. P. (2012). Lateral preference and schizotypy revisited: comparison of handedness measurement and classification methods. Laterality, 17(2), 150168.

Lentini, E., Kasahara, M., Arver, S., \& Savic, I. (2012). Sex differences in the human brain and the impact of sex chromosomes and sex hormones. Cerebral Cortex. http:// dx.doi.org/10.1093/cercor/bhs222.

Lombardo, M. V., Ashwin, E., Auyeung, B., Chakrabarti, B., Taylor, K., Hackett, G., et al. (2012). Fetal testosterone influences sexually dimorphic gray matter in the human brain. Journal of Neuroscience, 32(2), 674680.

Lust, J. M., Geuze, R. H., Van de Beek, C., Cohen Kettenis, P. T., Bouma, A., \& Groothius, T. G. G. (2011). Differential effects of prenatal testosterone on lateralization of handedness and language. Neuropsychology, 25(5), 581589.

Macchi, M. M., \& Bruce, J. N. (2004). Human pineal physiology and functional significance of melatonin. Frontiers in Neuroendocrinology, 25(3 4), 177195.

McCarthy, M. I., Abecasis, G. R., Cardon, L. R., Goldstein, D. B., Little, J., Ioannidis, J. P. A., et al. (2008). Genome wide association studies for complex traits: consensus, uncertainty and challenges. Nature Reviews Genetics, 9(May), 356369.

McManus, I. C., Davison, A., \& Armour, A. L. (2013). Multilocus genetic models of handedness closely resemble single locus models in explaining family data and are compatible with genome wide association studies. Annals of the New York Academy of Sciences, 1288(Jun), 4858.

McManus, I. C., Moore, J., Freegard, M., \& Rawles, R. (2010). Science in the making: right hand, left hand. III: estimating historical rates of left handedness. Laterality, 15(1 2), 186208.

Medland, S. E., Duffy, D. L., Wright, M. J., Geffen, G. M., Hay, D. A., Levy, F., et al. (2009). Genetic influences on handedness: data from 25,732 Australian and Dutch twin families. Neuropsychologia, 47(2), 330337.

Milenkovic, S., \& Dragovic, M. (2013). Modification of the Edinburgh handedness inventory: a replication study. Laterality, 18(3), 340348.

Milenković, S., Rock, D., Dragović, M., \& Janca, A. (2008). Seasons of birth and handedness in Serbian high school students. Annals of General Psychiatry, 7, 2. http://dx.doi.org/10.1186/ $1744859 \times 72$.

Nettle, D. (2003). Hand laterality and cognitive ability: a multiple regression approach. Brain and Cognition, 52(3), 390398.

Niebauer, C. L. (2004). Handedness and the fringe of consciousness: strong handers ruminate while mixed handers self reflect. Consciousness and Cognition, 13(4), 730745.

Oldfield, R. C. (1971). The assessment and analysis of handedness: the Edinburgh inventory. Neuropsychologia, 9(1), 97113.

Papadatou Pastou, M., Martin, M., Munafò, M. R., \& Jones, G. V. (2008). Sex differences in left handedness: a meta analysis of 144 studies. Psychological Bulletin, 134(5), 677699.

Peters, M., Reimers, S., \& Manning, J. T. (2006). Hand preference for writing and associations with selected demographic and behavioral variables in 255,100 subjects: the BBC internet study. Brain and Cognition, 62(2), 177189.

Preti, A., Lai, A., Serra, M., \& Zurrida, G. G. (2008). Mixed handedness prevails among children and adolescents with infantile asthma and diabetes. Pediatric Allergy and Immunology, 19(8), 769772.

Preti, A., Sisti, D., Rocchi, M. B., Busca, M., Vellante, M., Camboni, M. V., et al. (2011). Male female differences in left handedness in Sardinia, Italy. Laterality, 16(6), 737752. 
Stoyanov, Z., Nikolova, P., \& Pashalieva, I. (2011). Seasons of birth, Geschwind and Galaburda hypothesis, and handedness. Laterality, 16(5), 607619.

Tau, G. Z., \& Peterson, B. S. (2010). Normal development of brain circuits. Neuropsychopharmacology Reviews, 35, 147168.

Tonetti, L., Adan, A., Caci, H., Fabbri, M., \& Natale, V. (2012). Season of birth and handedness in young adults. Laterality, 17(5), 597601

Tran, U. S., Koller, I., Nader, I. W., Pietschnig, J., Schild, A. H. E., Stieger, S., et al. (2014). Lateral preferences for hand clasping and arm folding are associated with handedness in two large sample latent variable analyses. Laterality. http://dx.doi.org/ 10.1080/1357650X.2014.891607. advance online publication. Tran, U. S., Stieger, S., \& Voracek, M. (2014). Handedness and sex roles: mixed handers are less sex congruent stereotyped. Personality and Individual Differences, 66, 1013.

Vermunt, J. K., \& Magidson, J. (2005). Latent GOLD 4.0 user's guide. Belmont, Massachusetts: Statistical Innovations Inc.

Voracek, M., Reimer, B., Ertl, C., \& Dressler, S. G. (2006). Digit ratio (2D:4D), lateral preferences, and performance in fencing. Perceptual and Motor Skills, 103(2), 427446.

Wagenmakers, E. J., \& Forstmann, B. U. (2014). Rewarding high power replication research. Cortex, 51(February), 105106. 